\title{
Rythme et sens. À propos de quelques difficultés à traduire la poésie d'Henry Bauchau en allemand
}

L'or vert

$\grave{a} L$.

Je veux dans ma maison de sang

Un ami fort un ami lent

Tout près de l'âme reposant

Son île avec mon continent

Un rêveur un peintre un Flamand

Avec un pinceau d'or patient

Doux comme un saint très violent

Et qui me fait un cygne blanc

Un cygne noir comme un milan

Qui m'enlève et me donne au temps.
Grüngold

für L.

Ich will in meinem Haus aus Blut

Einen starken Freund einen rubigen Freund

Ganz nahe bei der Seele die ruht

Seine Insel mit meinem Kontinent

Ein Träumer ein Maler ein Flame

Mit einem Pinsel aus willigem Gold

Sanft wie ein heftiger Heiliger

Und der mir malt einen weißen Schwan

Einen Schwan so schwarz wie ein Weih

Der mich entfübrt und abgibt an die Zeit. ${ }^{\mathbf{2 0 0}}$

En 2001 a paru, sous le double titre Attentives - Lauschende, la première anthologie de poèmes d'Henry Bauchau en allemand - titre choisi par l'auteur. Le travail s'est avéré lent et long, la version allemande ne s'est jamais montrée définitive, telle une toile de Pénélope toujours à recommencer.

La difficulté majeure de la poésie bauchalienne, soumise à l'épreuve de la traduction, réside pour ces textes dans l'acte de rendre en allemand l'éblouissement du sens original français, produit par "l'écoute intérieure" et menant à un glissement de significations. C'est ainsi que le corps du poème se donne au lecteur comme une entité envoûtante, avec sa musicalité, ses sonorités, son rythme et le vécu de ses mots.

199 Henry Bauchau, Attentives - Lauschende. Poèmes choisis, traduits du français par Anne Neuschäfer, Aix-la-Chapelle, Shaker, 2001, p. 58-59. Les citations renverront directement à cette édition. 
Pour amener le lecteur en "arrière-pays» (terme cher à Yves Bonnefoy et Paul Willems) et afin de faire ressurgir avec force l'étrangeté de "la sourde oreille», le poète revisite depuis toujours la mythologie grecque et s'en inspire pour ses œuvres en prose comme en vers. Avant d'entreprendre la traduction des poèmes, il s'agit donc de saisir la relation de Bauchau au mythe et, pour cela, il convient de regarder de plus près les "constellations impérieuses " ${ }^{\mathbf{2 0 0}} \mathrm{du}$ mythe. La renaissance des personnages de la cité de Thèbes, du mythe des Labdacides, s'accomplit de façon magistrale dans le diptyque des deux romans Edipe sur la route et Antigone, où Henry Bauchau passe d'un premier sens du mythe - offrir un miroir et une exhortation à la mémoire des générations suivantes - à un deuxième, dérivé de la psychanalyse, où le mythe recouvre le domaine du rêve, du fantasme et du jaillissement de l'imaginaire. De cette manière, le romancier opère une superposition d'images, de dimensions et d'interprétations faisant surgir un autre CEdipe, né contre le verdict de la "non-naissance" comme le dit Jean Bollack ${ }^{\mathbf{2 0 1}}$ et appartenant au monde du désir, qui s'approprie son destin au moment où il semble l'avoir perdu aux yeux du monde. Ainsi, le protagoniste, symbole de l'anti-héros au départ, puisque condamné à la non-existence, incarne-t-il à la fin de son parcours les potentialités de ceux qui n'ont droit à rien et devient un exclu de la société, porteur cependant d'espérance. L'Edipe chez Bauchau naît d'un rapport à la fois historique, anthropologique, psychanalytique et existentiel que le poète établit par «l'écoute intérieure» du mythe avec lequel il entre en contact par l'imagination profonde. C'est de là que surgit alors un «vers donné», selon l'expression de Valéry, soit chez Bauchau un "son de voix", une "image "202 qu'il s'agit de capter et de transposer en langage rythmé - en premier lieu -, avant de lui donner un corps dans le récit en prose:

\section{Edipe à Colone}

J'ai vécu, j'ai connu d'un sauvage sommeil Le corps vertigineux des anciens labyrinthes

L'autel où j'ai brûlé sur tes falaises peintes L'or aigu et le sang des animaux vermeils

J'ai creusé dans ton lit le lit de ma rivière Le seuil vertigineux de ton aspérité Et j'annonce les feux de la nativité Qui feront déborder ta folie de lumière. ${ }^{\mathbf{2 0 3}}$

200 EE, p. 29.

201 Jean Bollack, La naissance d'Edipe, Paris, Gallimard, 1995.

202 EE, p. 29.

203 Attentives - Lauschende [...], p. 36-37. 
Le poème Edipe à Colone, daté de 1986, est antérieur à la publication du premier roman sur le destin du roi déchu de Thèbes. En se plaçant dans la perspective de celui qui a rejoint Colone (où CEdipe est accueilli par Thésée selon la légende), il jette un regard en arrière sur la vie passée de l'ancien tyran. Ce poème constitue ce que les Anciens appellent un accessus à la lecture du mythe thébain. C'est un texte bref, composé de deux quatrains aux alexandrins réguliers: ses rimes sont embrassées, comme s'il s'agissait de strophes de sonnet. Le lecteur peut noter l'absence de ponctuation, à l'exception du point final après le dernier vers. CEdipe parle à la première personne et entame son discours par une anaphore au passé composé, "j'ai vécu, j'ai connu» qui se poursuit dans les deux quatrains successifs. Plus loin, les strophes sont ponctuées par la récurrence de ces formes verbales à la première personne où se manifeste l'instance locutoire: «j'ai brûlé» et «j'ai creusé». Le changement du passé au présent intervient au septième vers où le «je» fait coïncider les deux dimensions temporelles; le dernier vers s'ouvre à l'avenir grâce à l'emploi du futur, au profit duquel la voix du «je» s'est effacée.

Si l'on relie les principaux substantifs entre eux, on retrouve, comme ramassé dans un filet de mots-clés, le parcours d'Cdipe: "Sommeil», "labyrinthes", "autel", "falaises" pour le premier quatrain, concluant triomphalement avec les couleurs de la sphinge, qui sont également celles de la reine, or et vermeil. Le «sommeil» évoque l'ignorance de la propre origine, les «labyrinthes" l'errance et le combat avec le Minotaure, l'«autel» la prophétie de Delphes et les "falaises" la haute muraille de Thèbes et/ou le précipice d'un mauvais choix imminent. Dans le deuxième quatrain, la répétition du substantif «lit» dans le cinquième vers fait allusion à l'inceste, ce que renforce le changement des pronoms possessifs passant de la première à la deuxième personne dans un enlacement inextricable ("ton lit le lit de ma rivière") de répétitions et de glissements sémantiques vers l'abreuvement du désir. "Le seuil [...] [de l']aspérité» indique l'orée du mystère dont le "je» énonciateur approche: l'«aspérité» l'amène à la découverte de ce qui était jusqu'alors ignoré, impliquant la notion de renoncement. Le revirement des deux derniers vers est présenté telle la chute d'un sonnet dans le deuxième tercet: «les feux de la nativité [...] feront déborder la folie de lumière", allusion directe à la naissance du Christ - folie peut-être plus grande que celle de la passion incestueuse - et qui enchâsse le débordement des amants inséparables dans un écrin de lumière, de pardon et de réconciliation.

La lecture littérale du mythe d'CEdipe se double d'une deuxième lecture, métaphorique, où le «je» locutoire coïncide avec le poète, décrivant par les images du mythe sa quête et l'enfantement du poème offert au monde. Le poète, arrivé au bout de son chemin, ressemble au tyran déchu: il s'est dépris de tout et arrive les mains nues à Colone, c'est-à-dire au bout de l'errance, au seuil de la forêt sacrée d'où son chant traduit l'universel, comme celui d'CEdipe qui devient le rhapsode de la Grèce. La rétrospective de cette deuxième lecture est métapoétique; elle exprime la vision du poète qui sonde son parcours, indiquant au lecteur les 
gisements de son imaginaire conceptuel et poétique, qui se place dans la continuation de Sophocle et reprend son flambeau:

\section{Sophocle sur la route}

à Bertrand Py

\section{Invitus Invitam}

Violemment, serviteur de la tragédie, tu as confié au temps

À l'immensité du théâtre

CEdipe l'aveuglant.

Tu l'as livré, tu l'as vendu

Sur la scène des grands brûlés

Où tes rois de mendicité portent leur couronne intérieure. ${ }^{204}$

\section{Sophokles unterwegs \\ für Bertrand Py}

\section{Invitus Invitam}

Ungestüm überaus hast du, der Tragödie Diener, ihn anvertraut der Zeit

Der Endlosigkeit des Runds

Ödipus, ihn blendend.

Ausgeliefert, ausgestellt hast du ibn

Auf der Bübne der großen Brennenden

Auf der deine Bettlerkönige die innere Krone tragen. ${ }^{205}$

Comment traduire un tel poème et le livrer à un public qui connaît tout au plus le roman Ödipus unterwegs, mais qui n'est pas familiarisé avec la poésie d'Henry Bauchau, ni avec ses journaux? Comment traduire l'alexandrin, sachant que la métrique en allemand suit l'accentuation et non le nombre des syllabes comme en français? Privilégier le sens, sachant que la connaissance de la mythologie grecque est liée en allemand aux plus grands poètes tels Hölderlin et Rilke et qu'il faudra donc trouver les mots justes, assurant la passerelle vers «l'arrière-pays » sans dénaturer Bauchau? Tout l'héritage de la philologie des lettres classiques, qui a connu son apogée à la fin du XIX ${ }^{\mathrm{e}}$ et au début du XX $\mathrm{XX}^{\mathrm{e}}$ siècle, est charrié dans le choix du vocabulaire. Ou suivre le rythme des images bauchaliennes, quitte à se laisser entraîner par les sonorités et heurter, le cas échéant, le sens qui se dérobera, 
glissera ailleurs, s'aliénera au texte original? Tout cela tient du défi et face aux dilemmes, nous avons opté pour l'écoute intérieure de chaque poème, soit traduire en suivant une impulsion guidée par la connaissance de l'œuvre du poète. Ce choix, il convient de le préciser, rend la traduction transitoire: elle n'est rien d'autre que l'effet d'un moment donné et peut évoluer autrement si l'écoute intérieure privilégie d'autres aspects en d'autres temps, ou, pour le dire avec les mots du poète:

\section{La circonstance}

Prisonnier d'un homme et d'un temps

Enfermé dans ma langue et le réseau de mes images

Je suis à tous, dit le poème, comme le ciel.

\section{Bewandtnis}

Häftling eines Mannes, einer Zeit

Verstrickt in Eigensprache und ins Netz der Eigenbilder

Gehör ich allen, sagt das Gedicht, wie der Himmel. ${ }^{\mathbf{2 0 6}}$

D'emblée, le choix a été fait de ne pas rendre le passé composé à la première personne ("Ich habe gelebt", "ich habe gekannt", "ich habe verbrannt", "ich habe gegraben") qui aurait introduit une fausse note épique typique du récit d'enfance, mais de rendre l'effet plus elliptique par l'emploi des participes "gelebt", "gekannt", préservant ainsi l'anaphore et son interpellation rythmique. "J'ai brûlé» a été substantivé par "Brandopfer", choix qui convient davantage en allemand à la construction du poème, rapprochant et condensant de cette manière "Altar" et "Brandopfer». Au deuxième quatrain, "j'ai creusé" a été remplacé par l'imparfait allemand "grub ich" qui est une forme de passé synthétique en allemand et a l'avantage d'éviter le "ich habe gegraben" qui appartient plus à la prose qu'au langage poétique. Au septième vers, la première personne a été conservée et traduite par "verkündige ich". Le choix de "verkündigen" plutôt que de "verkünden" s'explique par la proximité sémantique avec la "nativité" du Christ: les anges dans le ciel sur l'étable de Bethléem «verkündigen» la bonne nouvelle.

206 Ibid., p. 28-29. 
Un écueil de la traduction a été de rendre "vertigineux", difficulté accentuée par la double apparition de l'adjectif. Nous concédons que «schwindligmachend" n'est pas un mot joli, que l'on n'attend pas a priori dans un poème. Mais que faire? Traduit littéralement, "vertigineux" signifie "schwindlig", on dit par exemple "mir ist schwindlig» (j'ai le vertige). Dans le contexte du poème, le sens de l'adjectif semble porter davantage sur l'effet causé par "le corps" et «le seuil» et non sur le résultat, c'est-à-dire d'être pris de vertige; nous avons donc choisi de rendre le processus déclenché par la vue de ce qui rend "vertigineux" et qui peut se perpétuer. "Schwindligmachend" est un mot à la Brecht: il montre ce qui est en train de se passer, comme s'il rappelait un escalier en colimaçon dont on ne perçoit pas la fin. L'idée évoque l'infini face auquel CEdipe et le poète sont pris de peur. Ce n'est que grâce au chant et au poème qu'ils peuvent se débarrasser de cette angoisse. Le fait de souffrir de vertige est donc constitutif de cette poésie:

\title{
Hasardée dans le rêve
}

Hasardée dans le rêve avec tous ses gisants

Dans la vie qui s'écoule entre ses nouds coulants

J'ai vu la charité de la forme éclairant

Dans le monstre échoué le poème naissant. ${ }^{\mathbf{2 0 7}}$

\section{Achtlos im Traum}

\author{
Achtlos im Traum mit allem was rubt \\ Im Fluss des Lebens zwischen fein gleitenden Knoten \\ Sab ich im Glanz der Liebe erhellt die Form \\ Aus angelandetem Ungeheuer das werdende Gedicht. ${ }^{\mathbf{2 0 8}}$
}

Un deuxième écueil s'est présenté avec le verbe "déborder». Le dernier vers "[q]ui feront déborder ta folie de lumière" est obscur. Nous avons opté pour une traduction proche du sens littéral «über die Ufer gehen", en accentuant la présence $\mathrm{du}$ "je» locutoire par le choix de "treiben». Le vers rendu en allemand donne alors: "die deinen Wahn vor Licht über die Ufer treiben»" ${ }^{\mathbf{2 0 9}}$. Aujourd'hui nous aurions tendance à interpréter "déborder" comme "entgrenzen", afin de souligner l'idée que la folie (incestueuse et meurtrière) s'ouvre à l'infini, abandonne sa réclusion et y trouve enfin sa place et n'a donc plus besoin de recourir à des actions néfastes.

207 Ibid., p. 14. 208 Ibid., p. 15. 209 Ibid., p. 37. 
Ainsi, nous traduirions aujourd'hui le dernier vers par "die deinen Wabn im Licht entgrenzen", "vers donné» grâce à l'écoute intérieure dont le rythme correspond davantage au rythme du vers original.

\section{Ödipus in Kolonos}

Gelebt, gekannt in rohem Schlaf

Den schwindelmachenden Körper alter Labyrinthe

Den Altar, Brandopfer auf deinen gemalten Klippen

Von Scharfgold und Blut der feuerglühenden Tiere.

In dein Bett grub ich mein Flussbett

Schwindelmachende Schwelle deiner Herbe

Die Fackeln der Geburt verkündige ich

Die deinen Wabn im Licht entgrenzen.

Anne Begenat-Neuschäfer

RWTH Aachen 